\title{
Intención de práctica, satisfacción con la educación física y con la vida en función del género en estudiantes mexicanos y españoles Intention of practice, satisfaction with physical education and life by gender in Mexican and Spanish students \\ Raúl Fernández Baños \\ Universidad Autónoma de Baja California (México)
}

\begin{abstract}
Resumen. Los objetivos del presente estudio fueron: 1) Evaluar la relación entre la intención de práctica de actividad física en el tiempo libre con la satisfacción con la Educación Física y con la vida; y 2) analizar si existen diferencias en la intención de práctica de actividad física en el tiempo libre de los estudiantes, en la satisfacción con la Educación Física y con la vida en función del género y del país de procedencia. Participaron en el estudio 1137 estudiantes de educación secundaria obligatoria pertenecientes a centros públicos de España y México. Del total de la muestra, 457 fueron adolescentes mexicanos, de los cuales 246 eran chicas y 210 chicos. El resto de la muestra estuvo conformada por 680 adolescentes españoles, de los cuales 341 eran chicas y 339 chicos. Se utilizó un cuestionario compuesto por las siguientes escalas: Satisfacción con la materia de Educación Física, Satisfacción con la vida e Intención de Práctica de Actividad Física en el Tiempo Libre. Los análisis estadísticos que se realizaron fueron descriptivos, correlación de Spearman y la prueba de U de MannWithney, con el SPSS v.22. Los resultados revelaron diferencias significativas en función del sexo y en función del país. Adicionalmente, se encontró una relación positiva entre la satisfacción con la Educación Física con la intención de práctica y una relación negativa entre el aburrimiento con la educación física y la intención de práctica de actividad física en el tiempo libre.
\end{abstract}

Palabras clave: educación física, satisfacción, práctica futura, secundaria, adolescencia.

\begin{abstract}
The purposes of the present study were: 1) Assess the relationship between intention to practice physical activity in leisure time and physical education and life satisfaction; and 2) analyze the differences of the intention of practice physical activity in students' free time, satisfaction with physical education and life according to gender and origin. The participants of the study were 1137 Junior High School students belonging to public centers of Spain and Mexico. From the total sample, 457 were Mexican adolescents, of which 246 were girls and 210 boys. The rest of the sample was composed by 680 Spanish adolescents, 341 of which were girls and 339 boys. A questionnaire composed of the following scales was employed: Satisfaction with the Physical Education subject, Satisfaction with life and Intent of Practice of Physical Activity in Free Time. Descriptive statistical analysis with Spearman correlation and Mann-Whitney U test were applied according to the aims of the study and carried out with the SPSS v.22 software. The results showed significant differences in function of sex and country of origin. Additionally, a positive relationship between satisfaction with physical education and intention to practice, and a negative relationship between physical education boredom with the intention of practicing physical activity in free time were found.
\end{abstract}

Keywords: physical education, satisfaction, future practice, secondary, adolescence.

\section{Introducción}

Cada vez son más preocupantes los niveles de inactividad física en los adolescentes, siendo uno de los diez principales factores de riesgo de muerte en todo el mundo y responsable de enfermedades que causan morbilidad y mortalidad significativas según la Organización Mundial de la Salud (2016). El sedentarismo se ha relacionado con diferentes patologías que perjudican la calidad de vida del ser humano, asociándose con enfermedades como la depresión (Raudsepp \& Vink, 2019), diabetes (Baños, 2016), enfermedades cardiovasculares (Ruiz et al., 2016), obesidad (Tambalis, Panagiotakos, Psarra, \& Sidosis, 2019), trastorno bipolar (Vancampfort, et al., 2016) y la insatisfacción con la vida (Kleszczewska, Szkutnik, Siedlecka, \& Mazur, 2019).

El riesgo a desarrollar enfermedades relacionadas con el síndrome metabólico se relaciona con cantidades inadecuadas de actividad física en el tiempo libre y altos niveles de conductas sedentarias como cualquier conducta de vigilia caracterizada con un gasto energético d» 1.5 METs (Carson et al., 2016; Lee et al., 2012; Tremblay et al., 2016). Además, la problemática se acentúa cuando se observa que los niveles

Fecha recepción: 17-06-19. Fecha de aceptación: 23-10-19
Raúl Baños

raulfb89@gmail.com de actividad física en el tiempo libre disminuyen conforme los niños crecen hacia la etapa adolescente (Hagströmer, Kwak, Oja, \& Sjöström, 2015), convirtiéndose en un gran problema para la salud pública (Cliff et al., 2016).

Las cifras de inactividad física son alarmantes en adolescentes mexicanos y españoles, ya que los niveles de actividad físico-deportiva en su tiempo libre no son suficientes (Baños, Ruiz-Juan, Baena-Extremera, García-Montes, \& OrtizCamacho, 2018; Ruiz-Juan, Baena-Extremera, \& Baños, 2017; Vilchez-Conesa \& Ruiz-Juan, 2016). En esta línea, los adolescentes mexicanos se muestran aún menos activos que los españoles (Ruiz-Juan, et al., 2017), pudiendo incluso repercutir en la etapa adulta de los mexicanos (Zamarripa, RuizJuan, López, \& Baños, 2013), ya que se esperan peores tendencias conforme los adolescentes avancen en edad (Martínez-Gómez, et al., 2009), aumentando la probabilidad de sufrir diversas patologías (Oria, Zagalaz, López-Barajas, \& Aguilera, 2012), ocasionando con ello grandes costes económicos a la salud pública (Janssen, 2012).

Aumenta la preocupación si analizamos los niveles de actividad físicas en función del género (Vilchez-Conesa \& Ruiz-Juan, 2016). En adolescentes españoles, las mujeres obtienen valores bastantes más bajos que los hombres (de Looze, Elgar, Currie, Kolip, \& Stevens, 2019; Martínez-Baena, Mayorga-Vega, \& Viciana, 2016), perdurando esta diferencia también en la etapa adulta (López et al., 2016). En esta línea, 
aumenta la diferencia entre el género en adolescentes mexicanos, mostrándose los chicos como más activos (Wilkinson, Miller, Koehly, Daniel \& Forman, 2017), además de practicar actividad físico-deportiva de mayor intensidad (Cocca, Chmelik, Cocca, Espino, \& Rodenas, 2017). Como se puede observar, el panorama actual de adolescentes físicamente activos es inquietante en países como México y España, aunque no se encuentra suficiente literatura científica sobre la intención de practica futura en actividades físico-deportivas (Baños, Ortiz-Camacho, Baena-Extremera, \& TristánRodríguez, 2017).

La Teoría del Comportamiento Planeado de Ajzen (1991), es un constructo teórico socio-cognitivo que trata de explicar la «intención» del ser humano a desarrollar acciones futuras de actividad física, explicando los factores que promueven e influyen en la diversidad comportamental de los adolescentes hacia la practica físico-deportiva (Ajzen, 1991), habiéndose demostrado la validez y efectividad de la teoría (Ajzen, 2015). La literatura científica ha demostrado como esta teoría predice fuertemente la intención de realizar practica de actividad física en el tiempo libre (McEachan, Conner, Taylor, \& Lawton, 2011).

La satisfacción con la asignatura de Educación Física (EF), es un fuerte predictor de la intención de practica de actividad física en el tiempo libre de los estudiantes, produciendo mejoras en las actitudes y en el control del comportamiento del estudiante (Lwin y Malik, 2012). También, se relaciona con una mayor dedicación a la actividad física en horario extraescolar (Gabrielle, Gill, \& Claire, 2011; Kilpatrick, Hebert, \& Jacobsen, 2002), que a su vez aumenta la satisfacción con la vida (Videra-García \& Reigal-Garrido, 2013). Sin embargo, si el docente de EF no desarrolla una estrategia de motivación adaptada a sus estudiantes, aumentara la insatisfacción de éstos, disminuirá la importancia que le den a la materia y con ello la intención de práctica deportiva (BaenaExtremera, Granero-Gallegos, Sánchez-Fuentes, \& MartínezMolina, 2014: Taylor, Ntoumanis, Standage, \& Spray, 2010).

Como se pude leer en las anteriores líneas, la literatura científica ha evidenciado, por una parte, los bajos niveles de actividad física en el tiempo libre de los adolescentes mexicanos y españoles. Por otro lado, también ha quedado evidenciado la importancia que los estudiantes se sientan satisfechos con la materia de EF, para aumentar la intención de práctica deportiva y con ella, la satisfacción con la vida. Por ello, los objetivos de este estudio son los siguientes: 1) conocer la relación existente entre la intención de práctica de actividad física en el tiempo libre con la satisfacción con la EF y con la vida; y 2) analizar si existen diferencias en la intención de práctica de actividad física en el tiempo libre de los estudiantes, en la satisfacción con la EF y con la vida en función del género y del país de procedencia.

\section{Material y método}

\section{Diseño}

El presente estudio tiene un diseño descriptivo, transversal y no experimental (Sierra, 2001). Esta investigación se realizó de acuerdo con la Declaración de Helsinki de 1961 (revisada en Edimburgo en 2000).

\section{Participantes}

La muestra seleccionada fue un total de 1137 estudiantes de Educación Secundaria Obligatoria pertenecientes a centros públicos de España y México. Del total de la muestra, 457 fueron adolescentes mexicanos, de los cuales 246 eran $\operatorname{chicas}\left(\mathrm{M}_{\text {edad }}=14.01 \mathrm{DT}=.73\right)$ y $210 \operatorname{chicos}\left(\mathrm{M}_{\text {edad }}=14.21 \mathrm{DT}\right.$ $=.94)$. El resto de la muestra estuvo conformada por 680 adolescentes españoles, de los cuales 341 eran chicas $\left(\mathrm{M}_{\text {edad }}\right.$ $=14.82 \mathrm{DT}=1.46)$ y $339 \operatorname{chicos}\left(\mathrm{M}_{\text {edad }}=14.83 \mathrm{DT}=1.45\right)$.

\section{Procedimiento}

El estudio se dividió en dos fases siguiendo el mismo procedimiento en ambas. La primera fase se realizó en España y la segunda en México por el investigador principal. Primeramente, se concretó una cita con los directores de los centros de educación secundaria, dónde se les planteó e invitó a participar en el estudio. Los directores que rechazaron participar en el proyecto de investigación se descartaron $\mathrm{y}$ aquellos que accedieron se incluyeron en el estudio. Posteriormente, se solicitó a los padres/madres/tutores de los estudiantes, la firma de un consentimiento informado en el que se plasmaron los objetivos e intencionalidad del estudio. Los estudiantes que no tuvieron dicho consentimiento informado se descartaron del estudio. Se procedió a la recogida de datos informando previamente a los participantes del objetivo del estudio, la participación voluntaria y el tratamiento confidencial de sus repuestas puesto que el cuestionario era anónimo. Se les comunicó que no existían respuestas correctas ni incorrectas y se les pidió máxima sinceridad. Los cuestionarios, con una duración 15-20 minutos, se completaron en el aula estando siempre presente el mismo investigador que manifestó la posibilidad de consultarle cualquier duda durante el proceso. El protocolo del estudio fue aprobado por el Comité de Ética de la Universidad de Granada y por la Secretaria de Educación Pública de México y Universidad Autónoma de Baja California, México.

\section{Instrumentos} las:

El cuestionario estuvo formando por las siguientes esca-

Satisfacción con la materia de Educación Física (SATEF). Este instrumento fue traducido de la versión en original de Duda y Nicholls (1992), al castellano y adaptado a la materia de EF por Baena-Extremera, Granero-Gallegos, Bracho-Amador y Pérez-Quero (2012). Esta escala presenta ocho ítems que miden la satisfacción con las clases de EF, desglosándose en dos dimensiones: satisfacción/diversión (cinco ítems) y aburrimiento (ocho ítems). La escala estaba precedida por frase «Dinos tu grado de acuerdo o desacuerdo con la clase de Educación Física...». Las respuestas se recogen en escala tipo Likert desde 1 (muy en desacuerdo) hasta 5 (muy de acuerdo). Un ejemplo de ítem para de medir la satisfacción/diversión fue «Cuando estoy en clase de EF parece que el tiempo vuela», y de aburrimiento fue «En las clases de EF a menudo sueño despierto en vez de pensar en lo que hago realmente». Los valores de consistencia interna de las escalas para España fueron de .84 para la satisfacción y .71 para el aburrimiento, y en México de .78 para la satisfacción y .65 para el aburrimiento.

Satisfacción con la vida (SATV). Se usó la versión en 
castellano para evaluar la satisfacción con la vida de los adolescentes validado por Atienza, Pons, Balaguer y GarcíaMerita (2000), de la versión original de Diener et al., (1985). Este instrumento está compuesto por 5 ítems que miden la percepción que tienen los estudiantes sobre su satisfacción con la vida de forma global. La escala estaba precedida por la frase «Dinos tu grado de desacuerdo o acuerdo...». Las respuestas se recogen en escala tipo Likert desde 1 (muy en desacuerdo) hasta 5 (muy de acuerdo). Un ejemplo de ítem fue «Si pudiera vivir mi vida otra vez, la repetiría tal y como ha sido». Los valores de consistencia interna de escala en España fueron de .82, y para México de .74.

Intención de Práctica de Actividad Física en el Tiempo Libre (IP). Este instrumento fue traducido de la versión original de Chatzisarantis, Biddle y Meek (1997) basado en la obra de Ajzen y Madden (1986), al castellano por GraneroGallegos, Baena-Extremera, Pérez-Quero, Ortiz-Camacho y Bracho-Amador (2014). Este instrumento presenta tres ítems que miden la intención de los estudiantes de ser físicamente activos en su tiempo libre durante el periodo de un mes. La escala estaba precedida por frase «En mi tiempo libre fuera del instituto...». Las respuestas se recogen en escala tipo Likert desde 1 (muy en desacuerdo) hasta 7 (muy de acuerdo). Un ejemplo de ítem fue «Estoy decidido para hacer ejercicio físico». Los valores de consistencia interna de la escala en España fueron de .90, y para México de .86 .

\section{Análisis estadístico}

Inicialmente se llevó a cabo análisis descriptivos de cada subescala, los índices de asimetría, curtosis y el alfa de Cronbach para calcular la fiabilidad del instrumento. Posteriormente se realizó la prueba de Kolmogorov-Smirnov para estudiar la normalidad, comprobándose que los datos eran no paramétricos. Se realizó un análisis factorial confirmatorio (AFC) para estudiar la adecuación de dichos instrumentos a las muestras utilizadas en esta investigación y posteriormente se analizó la invarianza factorial. Tras los resultados obtenidos de la prueba de normalidad, se llevó a cabo un análisis de correlación de Spearman y la prueba de U de Mann-Withney para los contrastes de medidas por pares, tomando cada una de las dimensiones del cuestionario como variables dependientes y considerando el sexo y posteriormente el país, como variables de agrupación. Para todos los análisis se utilizó el paquete SPSS v.22 y el LISREL 8.80.

\section{Resultados}

\section{Estadística descriptiva}

En primer lugar en la Tabla 1, podemos observar los valores medios, de desviación típica, índices de asimetría, de consistencia interna y la prueba de normalidad de KolmorovSmirnov para cada factor de los instrumentos. Los valores medios más altos en la escala SATEF, fueron para los adolescentes españoles en la dimensión satisfacción/diversión $(\mathrm{M}=4.16 ; \mathrm{DT}=.87)$ y en la dimensión aburrimiento para los adolescentes mexicanos $(\mathrm{M}=2.00 ; \mathrm{DT}=1.05)$. Con respecto a la escala SATV, los valores medios más altos los obtuvieron los adolescentes mexicanos $(\mathrm{M}=3.59$; $\mathrm{DT}=.85)$. Para la escala de IP, los estudiantes españoles fueron los que obtuvieron los valores medios más altos $(\mathrm{M}=5.62 ; \mathrm{DT}=1.57)$. En cuan- to a la consistencia interna, casi todos los valores de alfa de Cronbach $(\alpha)$ están por encima de los valores aceptables según Dunn, Baguley y Brunsden (2014) y Hair, Black, Babin, y Anderson (2009). Aunque algunas dimensiones de la á hayan obtenidos valores por debajo de .70, el hecho de que presenten un número reducido de ítems por factor (como en el caso de la dimensión aburrimiento), hace que los valores de á puedan ser aceptables (Taylor, Ntoumanis, y Standage, 2008). Además, Nunnally (1967) ha mencionado que los valores de á de .60 e incluso .50, pueden ser suficientes para estudios que se encuentran en las primeras fases de investigación como el presente estudio. Los índices de asimetría deben ser próximos a $0 \mathrm{y}<2$, estando ligeramente por encima la dimensión aburrimiento en los estudiantes españoles. Los resultados de la prueba de Kolmogorov-Smirnov, indicaron que los datos presentaban una distribución no normal, por lo que el estudio de las correlaciones se utilizó el coeficiente de Spearman.

\section{Análisis factorial confirmatorio y de invarianza}

En la Tabla 2, se llevaron a cabo los AFC de cada uno de los instrumentos para ver que todos ellos eran válidos y fiables en la muestra que se quiere utilizar en esta investigación. Los resultados mostraron (Tabla 1) que son aceptables dentro de los límites establecidos en $\mathrm{x}^{2}$ 8gl (Bentler, 1989; Tabachnick \& Fidell, 2007), en GFI (Hooper, Coughlan \& Mullen, 2008), NFI, NNFI, CFI (Hu \& Bentler, 1995), y en RMSEA(Chen, Curran, Bollen, Kirby, \& Paxton, 2008; Cole \& Maxwell, 2003), a excepción de la escala SATV en la muestra española. Además, se analizó la invarianza factorial ( $\ddot{A}$ $\mathrm{CFI}$ ) en función del género del alumnado y del país para cada uno de los instrumentos. Los resultados mostraron adecuados índices de ajuste $(\ddot{\mathrm{A}} \mathrm{CFI}<0.01)$, tal y como indican diferentes estudios (Balaguer, Castillo \& Tómas, 1996; Duda, 1989; López-Walle, Tristán, Castillo, \& Balaguer, 2011).

\section{Análisis de correlación}

En relación al análisis de correlación (Tabla 3), se comprueba que la dimensión de satisfacción presenta valores negativos, altos y estadísticamente significativos con el aburrimiento y positivos con la intención de práctica futura de actividad física en ambos sexos de los adolescentes españoles. Las demás dimensiones obtuvieron relaciones moderadas estadísticamente significativas. En cuanto a los adolescentes mexicanos, las chicas obtuvieron valores positivos y estadísticamente significativos entre la intención de práctica con la satisfacción con la EF y con la vida, y negativa entre el aburrimiento en EF con la satisfacción en EF, y significativa moderada con la satisfacción con la vida. Sin embargo, los adolescentes mexicanos, en general obtuvieron valores de correlación más bajos que las chicas.

\section{Resultados según el sexo}

Para comprobar si existen diferencias en las dimensiones del estudio en función de la variable sexo, se utilizó la prueba estadística U de Mann Withney, como se puede observar en la Tabla 4. El sexo de los estudiantes se muestra como un factor determinante, obteniendo diferencias significativas. En México, los chicos presentan mayores niveles de SAT y IP que las chicas, ambas dimensiones con diferencias signi- 


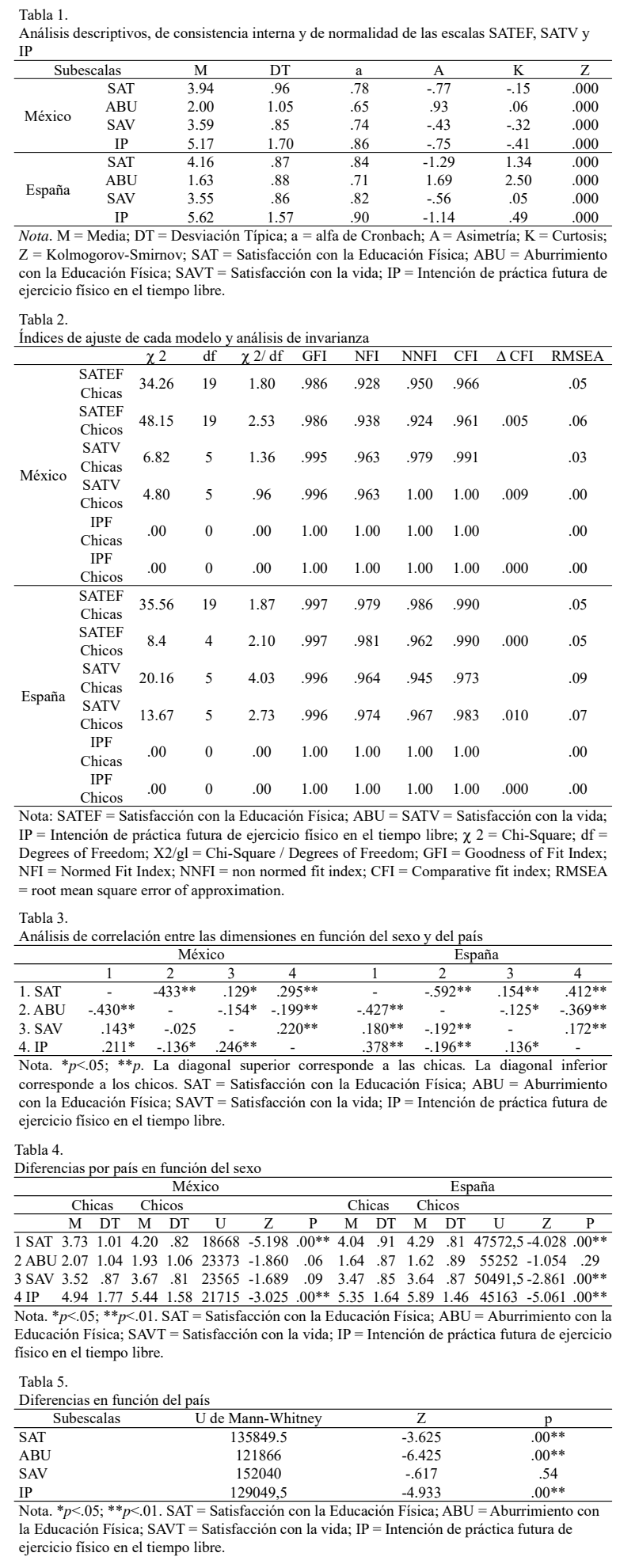

ficativas $(p<.01)$. Con respecto a los adolescentes españoles, los chicos presentan valores medios más altos en $S A T$, SATV y IP $(p<.01)$. No se encontraron diferencias en las dimensiones $A B U$ y $S A T V$ entre los adolescentes mexicanos, y tampoco en la escala $A B U$ en adolescentes españoles.

En cuanto a la diferencia entre países (ver Tabla 5), los estudiantes españoles obtuvieron niveles más altos de $S A T$ e IP $(p<.01)$, mientras que los estudiantes mexicanos reportaron tener mayores niveles de $A B U(p<.01)$. No se encontraron diferencias en las dimensiones SATV entre los adolescentes mexicanos y españoles.

\section{Discusión}

Los objetivos de esta investigación fueron: 1) conocer la relación existente entre la intención de práctica de actividad física en el tiempo libre con la satisfacción con la EF y con la vida; y 2) analizar si existen diferencias en la intención de práctica de actividad física en el tiempo libre de los estudiantes, en la satisfacción con la EF y con la vida en función del género y del país de procedencia. La importancia de este trabajo radica en el análisis actual sobre el bienestar subjetivo con la $\mathrm{EF}$ y con la vida en adolescentes mexicanos y españoles, y como estas variables se relacionan con la intención de realizar ejercicio físico en el tiempo libre, ya que ambos países obtienen niveles preocupantes de actividad física, enfatizándose más en los adolescentes mexicanos (Baños, et al., 2018).

El AFC realizado en cada muestra por separado, apoya la estructura factorial de las escalas, confirmándose la invarianza en la carga factorial. Con base a estos resultados, se pueden considerar que los instrumentos de este estudio son válidos para realizar estudios transculturales de comparación de medidas en función del género tal y como indican otros estudios (Balaguer et al., 1996; Duda, 1989; López-Walle et al., 2011).

En relación al primer objetivo, el presente trabajo halló relaciones significativas e indirectas entre la satisfacción con la EF con la variable de aburrimiento, y positivas con la intención de práctica de actividad física en el tiempo libre en los adolescentes españoles, siendo más fuerte la relación en las chicas que en los chicos. En cuanto a los adolescentes mexicanos, se encontraron relaciones similares con menor puntuación, sobre todo en los chicos. Resultados similares encontraron otros estudios, relacionando también la diversión o aburrimiento experimentado en las clases de EF con la intención de práctica en el tiempo libre de los adolescentes (Granero-Gallegos, Baena-Extremera, Sánchez-Fuentes, \& Martínez-Molina, 2014; Lwin \& Malik, 2012). Estos resultados podrían deberse al clima de aprendizaje que crea el docente en el aula, siendo más aconsejable los climas orientados al aprendizaje que los que se orientan a la demostración de habilidades (Baños, Baena-Extremera, Ortiz-Camacho, \& Tristán, 2016). De hecho, cuando un estudiante se divierte en la materia de EF, tiende a estar intrínsicamente motivado hacia la práctica deportiva, lo que supone un aumento de participación en las clases de EF e incluso, una mayor práctica de actividad física en el tiempo libre (Ntoumanis, 2005). La diferencia en los niveles de correlación entre los adolescentes españoles y los mexicanos, podría ser debido a la relación entre padres e hijos (Joyal-Desmarais, et al., 2019), siendo posible que los padres españoles motiven más intrínsicamente a sus hijos que los mexicanos.

En relación al segundo objetivo, se estudió si existían diferencias significativas en función del sexo. Por un lado, el presente estudio encontró diferencias significativas en las variables de satisfacción con la EF e intención de práctica de actividad física en el tiempo libre en los adolescentes mexicanos, obteniendo valores medios más altos los chicos que las chicas. Otras investigaciones hallaron resultados similares, donde los adolescentes mexicanos se mostraban más activos y con mayor satisfacción hacia la práctica deportiva que 
sus homólogas (Cocca, et al., 2017; Meneses-Montero \& Ruiz-Juan, 2017; Wilkinson, et al., 2017). Esta diferencia negativa hacia las chicas, podría deberse a distintos factores, entre los que se destacan la insatisfacción de la imagen corporal (Sámano, et al., 2015), la creencia que la actividad física no le reporta beneficios a su estado de salud (MenesesMontero \& Ruiz-Juan, 2017), entre otras variables. Sin embargo, se hace necesario seguir investigando sobre como los niveles de satisfacción con la EF se relacionan con la práctica deportiva de los adolescentes mexicanos, ya que no se encuentran suficientes estudios que confirmen dicha relación.

Por otro lado, se hallaron diferencias significativas en los estudiantes españoles, obteniendo los chicos valores medios más altos en satisfacción con la EF, satisfacción con la vida e intención de práctica futura que las chicas. Estos resultados podrían deberse a que las chicas están más orientadas a la búsqueda de la aprobación social de sus iguales que los chicos (Vilchez-Conesa \& Ruiz-Juan, 2016), aumentando su insatisfacción al focalizar sus objetivos en factores externos (Ruiz-Juan, Ortiz-Camacho, García-Montes, Baños, \& Baena-Extremera, 2018). Los resultados del presente estudio coinciden con los realizados por la literatura científica (Antunes, Serpa, \& Carita, 1999; Banos, Ortiz-Camacho, Baena-Extremera, \& Zamarripa, 2018; de Looze, et al., 2019; Martínez-Baena, et al., 2016). Es por esto, que algunos autores destacan la importancia de generar horas de calidad de $\mathrm{EF}$, a través de estrategias que repercutan en el aumento de la intención de práctica de actividad física (Ardoy, et al., 2010), en la satisfacción y motivación de los adolescentes hacia la EF (Granero-Gallegos, Baena-Extremera, Pérez-Quero, Ortiz-Camacho, \& Bracho-Amador, 2012).

En cuanto al análisis en función del país, se encontraron diferencias significativas con valores medios más altos de satisfacción con la EF y la intención de práctica de actividad física en el tiempo libre en los estudiantes españoles. Sin embargo, los adolescentes mexicanos obtuvieron valores medios más altos en aburrimiento con la EF, con respecto a los estudiantes españoles. Estas diferencias pueden deberse a que los estudiantes mexicanos se muestran más desmotivados y extrínsecamente motivados con la EF que los españoles (Ruiz-Juan, et al., 2018) o que perciben el clima de aprendizaje en las clases de EF más competitivo (RuizJuan, Baños, Fuentesal-García, García -Montes, \& BaenaExtremera, 2019). Los insuficientes niveles actuales de actividad física en adolescentes mexicanos (Baños, et al., 2018; Ruiz-Juan, et al., 2017; Wilkinson, et al., 2017), unidos a los resultados de este estudio sobre la escasa intención de práctica de actividad física en el tiempo libre, son indicadores negativos de la salud física y mental de éstos; aumentando incluso el riesgo de suicidio, siendo más preocupante en el género femenino (Hidalgo-Rasmussen \& Hidalgo-SanMartin, 2015), viéndose disminuido este indicador cuando se práctica actividad física en la cultura mexicana (HidalgoRasmussen, Ramírez-López, \& San-Martín, 2013). Además, preocupa los altos niveles de aburrimiento en el aula de EF, ya que el aburrimiento se relaciona con menor satisfacción con la vida (Scharenberg, 2016), con peores relaciones sociales entre los estudiantes (Persson, Haraldsson, \& Hagquist, 2016) y con comportamientos disruptivos (Olweus

\section{\&Breivik, 2014).}

\section{Conclusiones}

A modo de conclusión, este trabajo muestra que existen relaciones entre la satisfacción que los adolescentes mexicanos y españoles experimentan en las clases de EF, con la satisfacción con la vida y la intención de práctica de actividad física en el tiempo libre, mostrando relaciones más sólidas en los adolescentes españoles y en el sexo femenino. Cabe mencionar que los resultados de ambos países, mostraron menores niveles medios en todas las variables, por parte de las chicas. También destacar las diferencias entre ambos países, mostrando México niveles más bajos de satisfacción con la $\mathrm{EF}$ e intención de práctica futura y niveles más altos en aburrimiento con la EF. Estos datos complementan a la literatura científica, que ya ha demostrado los bajos niveles de actividad física en ambos países, aumentando la preocupación con los resultados del presente trabajo, ya que además, los niveles de práctica futura en el tiempo libre son bajos. Finalmente, como futuras líneas de trabajo, se podría estudiar cómo afecta en ambos países la figura del docente en el aumento de la satisfacción y la motivación hacia la práctica deportiva.

\section{Referencias}

Ajzen, I. \& Madden, T. (1986). Prediction of goal-directed behaviour: Attitudes, intentions and perceived behavioural control. Journal of Experimental Social Psychology, 22, 453-474. https://oi.org/ 10.1016/0022-1031(86)90045-4

Ajzen, I. (1991). The theory of planned behavior. Organizational behavior and human decision processes, 50(2), 179-211. https:/ /doi.org/10.1016/0749-5978(91)90020-T

Ajzen, I. (2015). The theory of planned behaviour is alive and well, and not ready to retire: a commentary on Sniehotta, Presseau, and Araújo-Soares. Health psychology review, 9(2), 131-137. https://doi.org/10.1080/17437199.2014.883474

Antunes, I., Serpa, S., \& Carita, I. (1999). Liderazgo y satisfacción en la educación física. Revista de Psicología del Deporte, 8(1), 0147-162.

Ardoy, D. N., Fernández-Rodríguez, J. M., Chillón, P., Artero, E. G., España-Romero, V., Jiménez-Pavón, D., ... \& Ortega, F. B. (2010). Educando para mejorar el estado de forma física, estudio EDUFIT: antecedentes, diseño, metodología y análisis del abandono/adhesión al estudio. Revista Española de Salud Pública, 84(2), 151-168. https://doi.org/10.1590/S113557272010000200004

Atienza, F. L., Pons, D., Balaguer, I., \& García-Merita, M. (2000). Propiedades psicométricas de la Escala de Satisfacción con la Vida en adolescentes. Psicothema, 12(2), 314-319.

Baena-Extremera, A., Granero-Gallegos, A., Bracho-Amador, C., \& Pérez-Quero, F. J. (2012). Spanish version of the sport satisfaction instrument (SSI) adapted to physical education. Revista de Psicodidáctica, 17(2), 377-395. https://doi.org/ 10.1387/RevPsicodidact.4037

Baena-Extremera, A., Granero-Gallegos, A., Sánchez-Fuentes, J. A., \& Martínez-Molina, M. (2014). Modelo predictivo de la importancia y utilidad de la Educación Física. Cuadernos de Psicología del Deporte, 14(2), 121-130. https://doi.org/10.4321/ S1578-84232014000200013

Balaguer, I., Castillo, I. \& Tomás, I. (1996). Análisis de las propiedades psicométricas del Cuestionario de Orientación al Ego y a la Tarea en el Deporte (teosq) en su traducción al castellano. 
Psicológica, 17, 71-81.

Baños, R. (2016). Prescripción del ejercicio físico en sujetos con diabetes mellitus tipo 2 y diabetes gestacional. Retos: Nuevas Tendencias en Educación Física, Deporte y Recreación, (29), 134-139.

Baños, R., Baena-Extremera, A., Ortiz-Camacho, M. M., \& Tristán J. L. (2016). Relación entre climas de aprendizajes, la importancia de la educación física y la intención de la práctica deportiva. En J. M. López-Walle et al. (Ed.), REDDECA. Contribuciones de la psicología para una Red de Deporte de Calidad (pp. 118-125). Nuevo León, México: Editorial Universitaria UANL.

Baños, R., Ortiz-Camacho, M. M., Baena-Extremera, A., \& TristánRodríguez, J. L. (2017). Satisfaction, motivation and academic performance in students of secondary and high school: background, design, methodology and proposal of analysis for a research paper. Espiral. Cuadernos del profesorado, 10(20), 40-50. https://doi.org/10.25115/ecp.v10i20.1011

Baños, R., Ortiz-Camacho, M. M., Baena-Extremera, A., \& Zamarripa, J. (2018). Efecto del género del docente en la importancia de la Educación Física, clima motivacional, comportamientos disruptivos, la intención de práctica futura y rendimiento académico. Retos: Nuevas Tendencias en Educación física, Deporte y Recreación, (33), 252-257.

Baños, R., Ruiz-Juan, F., Baena-Extremera, A., García-Montes, M., \& Ortiz-Camacho, M. (2018). Leisure-time physical activity in relation to the stages of changes and achievement goals in adolescents: Comparative study of students in Spain, Costa Rica, and Mexico. Sustainability, 10(7), 2581-2594. https://doi.org/10.3390/su10072581

Bentler, P. M. (1989), EQS Structural Equations Program Manual, BMDP, Los Angeles, CA.

Carson, V., Hunter, S., Kuzik, N., Gray, C. E., Poitras, V. J., Chaput, J. P., ... \& Kho, M. E. (2016). Systematic review of sedentary behaviour and health indicators in school-aged children and youth: an update. Applied Physiology, Nutrition, and Metabolism, 41(6), S240-S265. https://doi.org/10.1139/apnm2015-0630

Chatzisarantis, N. L., Biddle, S. J., \& Meek, G. A. (1997). A self determination theory approach to the study of intentions and the intention-behaviour relationship in children's physical activity. British Journal of Health Psychology, 2(4), 343-360. https://doi.org/10.1111/j.2044-8287.1997.tb00548.x

Chen, F., Curran, P. J., Bollen, K. A., Kirby, J., \& Paxton, P. (2008). An empirical evaluation of the use of fixed cutoff points in RMSEA test statistic in structural equation models. Sociological Methods and Research, 36, 462-494. https://doi.org/10.1177/ 0049124108314720

Cliff, D. P., Hesketh, K. D., Vella, S. A., Hinkley, T., Tsiros, M. D., Ridgers, N. D., ... \& Plotnikoff, R. C. (2016). Objectively measured sedentary behaviour and health and development in children and adolescents: systematic review and meta analysis. Obesity Reviews, 17(4), 330-344. https://doi.org/10.1111/ obr. 12371

Cocca, A., Chmelík, F., Cocca, M., Espino, F. D., \& Ródenas, L. T. (2017). Psychological, social and environmental predictors of physical activity in Mexican adolescents. Health Problems of Civilization, 11(3), 125-134. https://doi.org/10.5114/ hpc.2017.70524

Cole, D. A. \& Maxwell, S. E. (2003). Testing mediational models with longitudinal data: questions and tips in the use of structural equation modeling. Journal of Abnormal Psychology, 112, 558577. https://doi.org/10.1037/0021-843X.112.4.558

de Looze, M., Elgar, F. J., Currie, C., Kolip, P., \& Stevens, G. W. (2019). Gender inequality and sex differences in physical fighting, physical activity, and injury among adolescents across 36 countries. Journal of Adolescent Health, 64(5), 657-663. https://doi.org/10.1016/j.jadohealth.2018.11.007

Duda, J. L. (1989). Relationship between task and ego orientation and the perceived purpose of sport among high-school athletes. Journal of Sport \& Exercise Psychology, 11, 318-335. https:// doi.org/10.1123/jsep.11.3.318

Duda, J. L., \& Nicholls, J. G. (1992). Dimensions of achievement motivation in schoolwork and sport. Journal of educational psychology, 84(3), 290-299. https://doi.org/10.1037/00220663.84.3.290

Dunn, T. J., Baguley, T., \& Brunsden, V. (2014). From alpha to omega: A practical solution to the pervasive problem of internal consistency estimation. British Journal of Psychology, 105(3), 399-412. https://doi.org/10.1111/bjop.12046

Gabrielle, J., Gill, D., \& Claire, E. (2011). The roles of want to commitment and have to commitmentin explaining physical activity behavior. Journal of Physical Activity and Health, 8 , 420-428.

Granero-Gallegos, A., Baena-Extremera, A., Pérez-Quero, F. J., Ortiz Camacho, M. M., \& Bracho-Amador, C. (2014). Validación española del Intention to partake in leisure-time physicalactivity. Retos. Nuevas tendencias en Educación Física, Deportes y Recreación, 26, 40-45.

Granero-Gallegos, A., Baena-Extremera, A., Pérez-Quero, F. J., Ortiz-Camacho, M. M., \& Bracho-Amador, C. (2012). Analysis of motivational profiles of satisfaction and importance of physical education in high school adolescents. Journal of Sports Science \& Medicine, 11(4), 614-623.

Granero-Gallegos, A., Baena-Extremera, A., Sánchez-Fuentes, J. A., \& Martínez-Molina, M. (2014). Perfiles motivacionales de apoyo a la autonomía, autodeterminación, satisfacción, importancia de la educación física e intención de práctica física en tiempo libre. Cuadernos de Psicología del Deporte, 14(2), 5970. https://doi.org/10.4321/S1578-84232014000200007

Hagströmer, M., Kwak, L., Oja, P., \& Sjöström, M. (2015). A 6year longitudinal study of accelerometer-measured physical activity and sedentary time in Swedish adults. Journal of Science and Medicine in Sport, 18(5), 553-557. https://doi.org/10.1016/ j.jsams.2014.07.012

Hair, J., Black, W. C., Babin, B. J., \& Anderson, R. E. (2009). Multivariate data analysis. New York: Pearson Prentice Hall.

Hidalgo-Rasmussen, C. A., Ramírez-López, G., \& San-Martín, H. (2013). Actividad física, conductas sedentarias y calidad de vida en adolescentes universitarios de Ciudad Guzmán, Jalisco, México. Ciência \& Saúde Coletiva, 18, 1943-1952. https:/ /doi.org/10.1590/S1413-81232013000700009

Hidalgo-Rasmussen, C., \& Hidalgo-San-Martín, A. (2015). Comportamientos de riesgo de suicidio y calidad de vida, por género, en adolescentes mexicanos, estudiantes de preparatoria. Ciência \& Saúde Coletiva, 20,3437-3445. https:/ /doi.org/10.1590/1413-812320152011.18692014

Hooper,J. D., Coughlan, M.R., \& Mullen, M. (2008). Structural equation modeling: Guidelines for determining model fit. The Electronic Journal of Business Research Methods, 6, 53-60.

Hu, L.-T., \& Bentler, P. (1995). Evaluating model fit. In R. H. Hoyle (Ed.), Structural Equation Modeling. Concepts, Issues, and Applications (pp.76-99). London: Sage.

Joyal-Desmarais, K., Lenne, R. L., Panos, M. E., Huelsnitz, C. O., Jones, R. E., Auster-Gussman, L. A., ... \& Rothman, A. J. (2019). Interpersonal effects of parents and adolescents on each other's health behaviours: a dyadic extension of the theory of planned behaviour. Psychology \& health, 34(5), 569-589. https://doi.org/10.1080/08870446.2018.1549733

Kleszczewska, D., Szkutnik, A. M., Siedlecka, J., \& Mazur, J. (2019). Physical Activity, Sedentary Behaviours and Duration of Sleep as Factors Affecting the Well-Being of Young People against the Background of Environmental Moderators. International journal of environmental research 
and public health, 16(6), 915-929. https://doi.org/10.3390/ ijerph16060915

Kilpatrick, M., Hebert, E., \& Jacobsen, D. (2002). Physical activity motivation. A practitioner's guido to self-determination theory. Journal of Physical Education, Recreation and Dance, 74(4), 3641.

Lee, I. M., Shiroma, E. J., Lobelo, F., Puska, P., Blair, S. N., Katzmarzyk, P. T., \& Lancet Physical Activity Series Working Group. (2012). Effect of physical inactivity on major non-communicable diseases worldwide: an analysis of burden of disease and life expectancy. The lancet, 380(9838), 219-229. https://doi.org/10.1016/S01406736(12)61031-9

López, R. C., Santos, M. A., Navarro, A. V., Arévalo, J. M., García, F., \& Latorre,P. Á. (2016). Determinantes sociodemográficos y nivel de actividad física en la población de la provincia de Jaén mayor de 18 años. Retos: Nuevas Tendencias en Educación Física, Deporte y Recreación, (29), 13-16.

López-Walle, J., Tristán, J., Castillo, I. T. I., \& Balaguer, I. (2011). Invarianza factorial del TEOSQ en jóvenes deportistas mexicanos y españoles. Revista Mexicana de Psicología, 28(1), 53-61.

Lwin, M. O., \& Malik, S. (2012). The efficacy of exergames-incorporated physical education lessons in influencing drivers of physical activity: a comparison of children and pre-adolescents. Psychology of Sport and Exercise, 13(6), 756-760.

Martínez-Baena, A. C., Mayorga-Vega, D., \& Viciana, J. (2016). Relación de los niveles de actividad física con el género y el perfil de riesgo cardiovascular en adolescentes granadinos. Implicaciones didácticas para la educación física. Profesorado: Revista de curriculum y formación del profesorado, 20(1), 265-285.

McEachan, R. R. C., Conner, M., Taylor, N. J., \& Lawton, R. J. (2011). Prospective prediction of health-related behaviours with the theory of planned behaviour: A meta-analysis. Health Psychology Review, 5(2), 97-144. https://doi.org/10.1080/ 17437199.2010 .521684

Meneses-Montero, M. M., \& Ruiz-Juan, F. (2017). Estudio longitudinal de los comportamientos y el nivel de actividad físico-deportiva en el tiempo libre en estudiantes de Costa Rica, México y España. Retos: Nuevas Tendencias en Educación Física, Deportey Recreación,(31), 219-226.

Ntoumanis, N. (2005). A prospective study of participation in optional school physical education using a self-determination theory framework. Journal of Educational Psychology, 97, 444-453. https:/ /doi.org/10.1037/0022-0663.97.3.444

Nunnally, J. C. (1967). Psychometric theory. New York, NY, US: McGraw-Hill.

Olweus, D., \& Breivik, K. (2014). Plight of Victims of School Bullying: The Opposite of Well-Being. In A. Ben-Arieh, F. Casas, I. Frønes, \& J. E. Korbin (Eds.), Handbook of Child Well-Being SE - 90 (pp. 2593-2616). Netherlands: Springer. https://doi.org/10.1007/97890-481-9063-8_100

Organizacion Mundial de la Salud [OMS]. (2016). Physical activity. Factsheet. Retrieved December 7, 2016 from http://www.who.int/ mediacentre/factsheets/fs385/en/.

Oria, H. M., Sánchez, M. L. Z., López-Barajas, D. M., \& Aguilera, S. C. (2012). Prevención de la obesidad infantil a través de una motivación intrínseca hacia la práctica de actividad física. Retos: Nuevas tendencias en Educación Física, Deporte y Recreación, (22), 49-52.

Persson, L., Haraldsson, K., \& Hagquist, C. (2016). School satisfaction and social relations: Swedish school children'simprovement suggestions. International Journal of Public Health, 61(1), 83-90. https://doi.org/10.1007/s00038-015-0696-5

Ruiz, J. R., Cavero-Redondo, I., Ortega, F. B., Welk, G. J., Andersen, L. B., \& Martinez-Vizcaino, V. (2016). Cardiorespiratory fitness cut points to avoid cardiovascular disease risk in children and adolescents; what level of fitness should raise a red flag? Asystematic review and meta-analysis. British Journal Sports Medicine, 50(23), 1451-1458. https://doi.org/10.1136/bjsports-2015-095903

Ruiz-Juan, F., Baena-Extremera, A., \& Baños, R. (2017). Nivel de actividad deportiva en el tiempo libre desde las etapas de cambio y motivación en estudiantes de Costa Rica, México y España. Cuadernos de Psicología del Deporte, 17(2), 53-64.

Ruiz-Juan, F.; Baños, R.; Fuentesal-García, J.; García-Montes, E. \& Baena-Extremera, A (2019). Análisis transcultural del clima motivacional en alumnado de Costa Rica, México y España. Revista Internacional de Medicina y Ciencias de la Actividad Física yel Deporte, 19 (74) 351-369.

Ruiz-Juan, F.; Ortiz-Camacho, M.M.; García-Montes, M.E.; BaenaExtremera, A. \& Baños, R. (2018). Predicción transcultural del clima motivacional en educación física. Revista Internacional de Medicina y Ciencias de la Actividad Física y el Deporte. 18 (69), 165-183. https://doi.org/10.15366/rimcafd2018.69.011

Sámano, R., Rodríguez-Ventura,A. L., Sánchez-Jiménez, B., Martínez, G., Ytelina, E., Noriega, A., ... \& Nieto, J. (2015). Satisfacción de la imagen corporal en adolescentes y adultos mexicanos y su relación con la autopercepción corporal y el índice de masa corporal real. Nutrición Hospitalaria, 31(3), 1082-1088.

Scharenberg, K. (2016). The interplay of social and ethnic classroom composition, tracking, and gender on students' school satisfaction. Journal of Cognitive Education and Psychology, 15(2), 320-346. https://doi.org/10.1891/1945-8959.15.2.320

Sierra, R. (2001). Técnicas de investigación social. Teoría y ejercicios. Madrid: ed. Paraningo Thomson Learning.

Tabachnick, B. G \& Fidell,L. S. (2007).Using multivariate statistics, 5. ed. New York: Allyn and Bacon.

Tambalis, K. D., Panagiotakos, D. B., Psarra, G., \& Sidossis, L. S. (2019). ConcomitantAssociations between Lifestyle Characteristics and Physical Activity Status in Children and Adolescents. Journal of Research in Health Sciences, 19(1), 1-7.

Taylor, I. M., Ntoumanis, N., \& Standage, M. (2008). A selfdetermination theory approach to understanding the antecedents of teachers' motivational strategies in physical education. Journal of Sport Exercise Psychology, 30, 75-94. https://doi.org/10.1123/ jsep.30.1.75

Tremblay, M. S., Aubert, S., Barnes, J. D., Saunders, T. J., Carson, V., Latimer-Cheung, A. E., ... \& Chinapaw, M. J. (2017). Sedentary behavior research network (SBRN)-terminology consensus project process and outcome. International Journal of Behavioral Nutrition and Physical Activity, 14(1), 75. https://doi.org/10.1186/s12966017-0525-8

Vancampfort, D., Firth, J., Schuch, F., Rosenbaum, S., De Hert, M., Mugisha, J., ... \& Stubbs, B. (2016). Physical activity and sedentary behavior in people with bipolar disorder: a systematic review and meta-analysis. Journal of affective disorders, 201, 145-152. https:/ /doi.org/10.1016/j.jad.2016.05.020

Videra-García,A., \& Reigal-Garrido, R. E. (2013).Autoconcepto físico, percepción de salud y satisfacción vital en una muestra de adolescentes. Anales de Psicología, 29(1), 141-147.https://doi.org/ 10.6018/analesps.29.1.132401

Vilchez-Conesa, M. P., \& Ruiz-Juan, F. (2016). Clima motivacional en Educación Física y actividad físico-deportiva en el tiempo libre en alumnado de España, Costa Rica y México. Retos: Nuevas Tendencias en Educación Física, Deporte y Recreación, (29), 195-200.

Wilkinson, A. V., Miller, E. E., Koehly, L. M., Daniel, C. R., \& Forman, M. R. (2017). Correlates of Physical Activity Differ by Sex and Country of Birth Among Mexican-Heritage Youth. Journal of immigrant and minority health, 19(2), 246-253. https://doi.org/ 10.1007/s10903-016-0451-x

Zamarrima, J., Ruiz-Juan, F., López, J. M., \& Baños, R. (2013). Actividad e inactividad física durante el tiempo libre en la población adulta de Monterrey (Nuevo León, México). Retos. Nuevas tendencias en Educación Física, Deporte y Recreación, (24), 9196. 